\title{
Effects of Alternate Training for Limb Motor Skills on Brain-derived Neurotrophic Factor and Gait in Striatal Hemorrhage-induced Sprague-Dawley Rats
}

\author{
Dae-hwan Lee ${ }^{1}$, Jung-ho Lee ${ }^{2}$ and Youn-bum Sung ${ }^{3 *}$ \\ ${ }^{1}$ Dept. Physical Therapy, Daegu Univ., 201, Daegudae-ro, Jillyang-eup, Gyeongsan- \\ si, Gyeongsangbuk-do, 38453, Republic of Korea \\ ${ }^{2}$ Dept. Physical Therapy, Kyungdong Univ., Bongpo-ri, Toseong-myeon, Goseong- \\ gun, Gangwon-do, 219-705, Republic of Korea \\ ${ }^{3}$ (Corresponding Author), Dept. Physical Therapy, Daegu Univ., 201, Daegudae-ro, \\ Jillyang-eup, Gyeongsan-si, Gyeongsangbuk-do, 38453, Republic of Korea \\ Idhlee8510@naver.com, ${ }^{2}$ ljhcivapt@naver.com, ${ }^{3}$ playeryoon@naver.com
}

\begin{abstract}
In the brain hemorrhage causes damage to the patient's brain, and the impairment of neurotrophic factors and cognitive and motor disorders result in restrictions on daily and functional activities. In this study, we conducted abutment and familiar exercise in striatal hemorrhagic SD rat to compare neurotrophic factors and functional activities. The manifestation of BDNF in the brain of white mice was $107.08 \pm 1.85$ in the group that conducted the abutment movement and $104.97 \pm 0.85$ in the group that performed the familiar exercise, and was statistically significant. The Beam walking test scores were 4.530 .50 .52 points for the group that conducted the abutment movement and $4.80 \pm 0.41$ points for the group that performed the familiar movement, and statistically significant. It has been shown that alternating exercise can have a more positive effect on nerve recovery and motor function on striatal hemorrhage. This study suggests this alternative body motion pattern as a useful intervention for patients with cerebral hemorrhage.
\end{abstract}

Keywords: Striatal hemorrhage, BDNF, Beam walking test, Treadmill, Swimming

\section{Introduction}

Intracerebral hemorrhage, which accounts for $10 \%$ to $15 \%$ of stroke cases, occurs due to cerebrovascular diseases, chronic hypertension, and similar conditions [1]. Approximately $40 \%$ of patients with intracerebral hemorrhage die within six months after its onset, and only about $20 \%$ of these patients recover their abilities to perform Activities of Daily Living (ADLs) independently.

According to MacLellan, patients with intracerebral hemorrhage can develop cognitive and perceptive disorders as well as long-term motor skills and sensory disorders, causing limitations in ADLs and functional activities, significantly reducing the quality of life [2]. About $65 \%$ of intracerebral hemorrhage patients experience loss of their bodies' protective responses and proprioception, and their reduced muscle tension translates into instability and

Article history:

Received (March 5, 2020), Review Result (April 10, 2020), Accepted (May 18, 2020) 
decreased efficiency of motions [3]. Specifically, the connectivity between the hippocampus and the striatum is responsible for cognitive and motor control, and striatal impairment results in cognitive, motor, and ADL-related disorders [4].

Stroke patients' exercises have been applied in various ways. A study reported that exercising before stroke onset can prevent brain damage, and wheel running applied in animal models reduces the degrees of cerebral edema and infarction after the onset of cerebral infarction [5]. Wheel running before the onset of cerebral infarction lowers the blood-brain barrier penetrability and reduces the amount of the neurotransmitter glutamate, thereby decreasing neurotoxicity and impairment immediately after the onset [6]. These findings have led researchers to use running machines and instruments for wheel running or environmental strengthening as intervention tools [7]. These tools also improve the connectivity between motor areas during the learning of exercises [8][9]. In addition, the application of new exercises in patients with cranial nerve injuries is more effective than the continuance of their familiar exercises [10].

The brain's Nerve Growth Factors (NGFs) include the Brain-Derived Neurotrophic Factor (BDNF) and the insulin-like factor-1. These factors are closely linked with the recovery of the central nervous system and can be activated through a variety of physical exercises and activities [10]. According to previous research, these factors are involved in the survival, differentiation, and formation of nerve cells. Specifically, as a neurotrophic protein, the BDNF also exists in the adult brain; it is isolated from nerve cells, affects learning and memory, and functions as an important signal for the brain's neuroplasticity. Therefore, it is an important factor for brain recovery after brain damage [11]. The purpose of the present study is to compare the effects of steady training and alternate training on the BDNF and hand function in white rats with intracerebral hemorrhage.

\section{Research methods}

\subsection{Test animals}

In this study, an experiment was conducted using 16 eight-week-old Sprague-Dawley rats without clinical or neurological disorders. During the experiment, these rats were given light and dark cycles at 12 -hour intervals at room temperature $\left(23^{\circ} \mathrm{C} \pm 2^{\circ} \mathrm{C}\right)$ and $60 \% \pm 5 \%$ humidity. The experimental procedures complied with the Guide for the Care and Use of Laboratory Animals, issued by the Institute of Laboratory Animal Resources. The white rats had a five-day acclimation period before the experiment to become accustomed to the breeding environment.

In the control group $(n=8)$, the rats were first trained in wheel running for one week, and cerebral hemorrhage was then induced in them. Next, they had to perform wheel running again for two weeks. In the experimental group $(n=8)$, the rats were trained in wheel running for one week, and cerebral hemorrhage was then induced. This was followed by a two-week swimming training.

\subsection{Exercise methods}

Wheel running has widely been used as an effective exercise for patients with nerve injuries to practice rhythmic walking. Its advantage is that the amount of exercise can be easily calculated [12]. In this study, rats were conditioned to perform wheel running for 15 minutes once a day at $55 \%$ of the maximum $\mathrm{VO} 2$ and at a speed of $15 \mathrm{~m}$ per minute [9]. 
Electrical stimulations were applied at less than $2.0 \mathrm{~mA}$ to make the rats continue to exercise without breaks.

Swimming can be used to reduce patients' stress levels during training; its advantage is that greater resistance can be applied than that in wheel learning [13]. Moreover, it can train multiple animals at the same time. In this study, the rats performed a swimming exercise under no-load conditions for 15 minutes once a day. The water temperature was maintained at $33^{\circ} \mathrm{C}$ to $35^{\circ} \mathrm{C}$, and the water depth was set at $50 \mathrm{~cm}$ to prevent the rats from supporting their tails against the bottom [14].

\subsection{Brain-derived neurotrophic factor (BDNF)}

As a critical factor related to the recovery of the central nervous system, the BDNF controls cerebral development and neurogenesis; it also protects dopamine, cortexes, and motor nerves that prevent the occurrence of neurological disorders on the central nerve system. Thus, the BDNF is frequently expressed in the hippocampus and the cortex and involved in motor learning, memory, and long-term memory [11][15].

\subsection{Test methods}

\subsubsection{Beam walking test}

The beam walking test is used to evaluate the control of motor skills and the sensorimotor system. Repeated toe flexion and extension are highly important. Two weeks after the inducement of striatal hemorrhage, the rats were trained to walk on a wooden beam $(2.5 \times 2.5$ $\times 80 \mathrm{~cm}$ ), and their gait was then scored from 0 to 6 points. Each rat's performance was measured three times for comparison. The 0 score is the best performance with non slips and 6 score is unable to maintain the body on the beam for 10 seconds. The 6 is the worst score.

\subsubsection{BDNF immunohistochemistry}

Immunohistochemistry was employed to compare the levels of BDNF expression. Each rat was given general anesthesia with a $2 \mathrm{mg} / \mathrm{kg} 1: 1$ mixture of Zoletil and Rompun. The rat's body was drained of blood through blood perfusion using a $0.9 \% \mathrm{NaCl}$ solution, and the body was fixed in a $4 \%$ para-formaldehyde solution to extract its brain tissue. After 24 hours, the extracted brain tissue was frozen in a cryocooler at $-30^{\circ} \mathrm{C}$ and then severed into $30 \mu \mathrm{m}$-thick brain slices using a cryostat. After the brain slices were mounted on a slide and the first antibody was applied to them, they were kept at $4^{\circ} \mathrm{C}$ for 24 hours. The slices were then immersed in 0.01M Phosphate-Buffered Saline (PBS) for 10 minutes three times. After the application of the secondary antibody, these slices were preserved at room temperature for two hours. Next, the slices were again immersed in 0.01M PBS for 10 minutes three times and then underwent a dehydration process using ethanol and xylene. Finally, the resulting slices were mounted with a cover glass.

\subsection{Data processing}

Photos were taken for a comparison of the BDNF expression levels by using an Axiophot light microscope (Carl Zeiss, Göttingen, Germany) with an installed charge-coupled device (software: Optimas 6.5, Cyber Metrics, Scottsdale, Arizona). For each rat, 30 brain slices were used. The photos were calibrated using Adobe Photoshop version 8.0 and analyzed through the program NIH Image 1.59. The data were analyzed using SPSS version 20.0 for 
Windows. The analysis results were indicated as mean \pm standard deviation, and each group was compared through an independent t-test. In this paper, the independent t-test was used for comparison of the two groups. The independent variable used the alternate exercise, and as a dependent variable, the degree of expression of BDNF was compared to confirm the histological change, and the beam walking test was conducted to check the ability of the upper and lower extremities to perform their functions. To compare the data, descriptive statistics was conducted and the mean was used as a representative value, and standard deviation was used to check the deviation of the data.

\section{Results}

\subsection{Beam walking test}

The beam walking test was conducted to evaluate the effects of alternate exercises on sensory function, motor skills, and balance ability. The control and experimental groups recorded $4.80 \pm 0.41$ and $4.53 \pm 0.52$ points, respectively. The two groups showed a statistically significant difference.

Table 1. Beam walking test Score (0 to 6 score)

\begin{tabular}{|c|ccc|c|c|}
\hline Group & Mean & \pm & SD & t-value & $p$ \\
\hline Control & 4.80 & \pm & 0.41 & \multirow{2}{*}{10.12} & $.000 * * *$ \\
\hline Experiment & 4.53 & \pm & 0.52 & \\
\hline
\end{tabular}

* Control group: exercise treadmill both before onset and after onset

* Experiment group: exercise treadmill before onset and swimming after onset

$*$ Mean $\pm S D$ (standard deviation)

$* p<.05 *,<.01 * *,<.001 * * *$

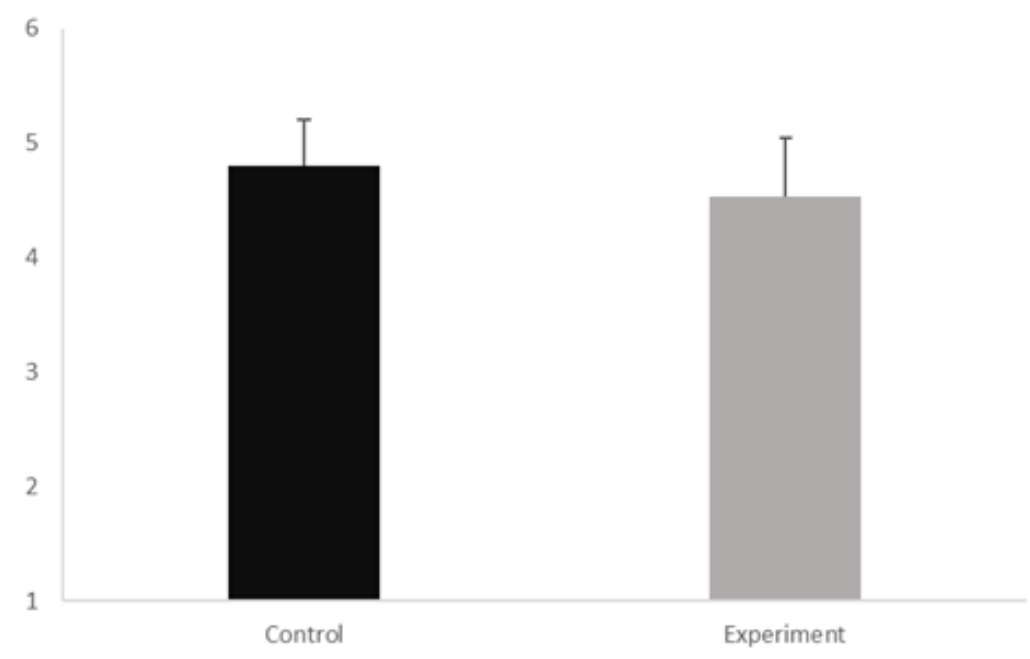

Figure 1. Graph of beam walking test score (0 to 6 score)

\subsection{BDNF immunohistochemistry}

BDNF immunohistochemistry was conducted to assess the effects of alternate exercises on the expression of NGFs. The control and experimental groups registered $104.97 \pm 0.85$ and 
$107.08 \pm 1.85$ points, respectively. A statistically significant difference was observed between the two groups.

Table 2. Immunohistochemistry expression density of BDNF in striatum

\begin{tabular}{|c|ccc|c|c|}
\hline Group & Mean & \pm & SD & t-value & $p$ \\
\hline Control & 104.97 & \pm & 0.85 & \multirow{2}{*}{$* 000 * * *$} \\
\hline Experiment & 107.08 & \pm & 1.85 & 36.64 & .004 \\
\hline
\end{tabular}

* Control group: exercise treadmill both before onset and after onset

* Experiment group: exercise treadmill before onset and swimming after onset

* Mean $\pm S D$ (standard deviation)

$* p<.05 *,<.01 * *,<.001 * * *$

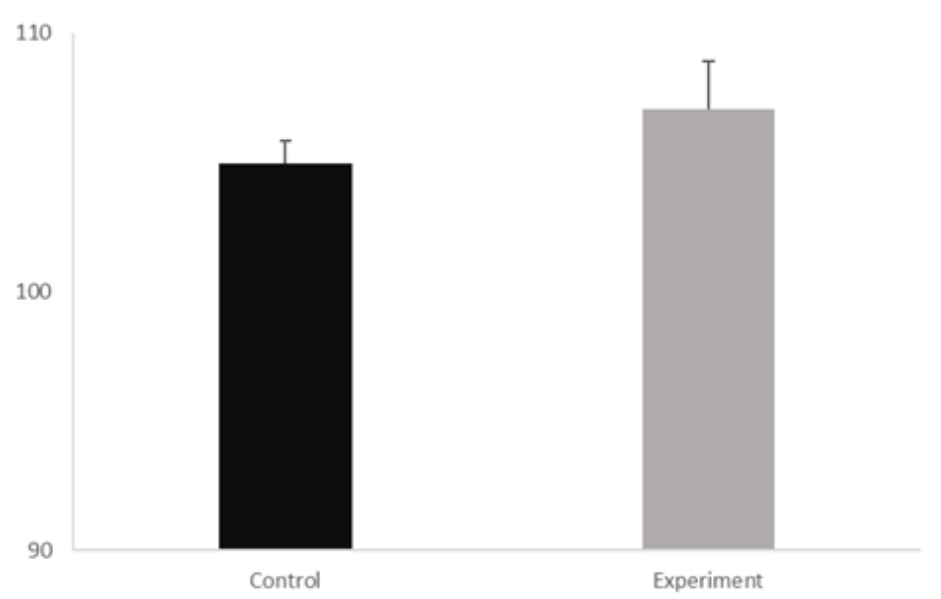

Figure 2. Graph of immunohistochemistry expression density of BDNF in striatum

\section{Discussion}

This study was based on the theory of rehabilitation that the body's exercise helps the brain to activate, and that training by alternating exercise will help accelerate brain reorganization and be more effective in improving function.

Brain-damaged patients generally exhibit weakened muscle strength, cognitive imbalance, and proprioceptive dysfunction, which subsequently cause disorders in balance, coordination, and functional movement. This study examined the effects of varied application patterns for exercises on the recovery of motor skills and neurotrophic factors in white rats with intracerebral hemorrhage. The effects of alternate exercises on stroke SD rats were investigated by comparing the group that continued a single exercise and the group that performed a different exercise after the inducement of the disease through an analysis of neurotrophic factors and behaviors.

A dominant portion of studies on cerebrovascular diseases are focused on medical domains, such as medication, surgery, and cell transplantation. Exercise interventions are confined to simple wheel running or swimming. The advantage of physical exercises in patients with cerebrovascular diseases is not only improved motor abilities but also lowered incidences of these diseases and mortality rates [16]. In the present study, the beam walking test was conducted to understand the clinical patterns of stroke-induced white rats and measure their sensory and motor abilities, balance, and control. The beam walking test is a highly effective testing method of evaluating the functions of patients with stroke [6]. 
The BDNF is a neurotrophic protein complex that induces neural development and functions. It secretes proteins in locations intended for the survival, differentiation, or growth of neurons [9][17][19]. Neurotrophic substances influence the formation of new blood vessels, the survival and differentiation of neurons, and the formation and plasticity of synapses [18, 19]. The BDNF is a key activator protein that stimulates neurogenesis and post-birth phases with the most dynamic control [15][19].Because the BDNF is vital for the protection and recovery of nerves against neurotoxins, increased BDNF cells markedly improve motor learning [18].

Stroke patients' disorders in functional activity are exhibited in the form of weakened upper and lower limb muscles, poor muscular endurance, and proprioceptive and cognitive disorders, which eventually undermine their motor skills, postural control, and balance ability [19]. The beam walking test evaluates coordination, motor performance, and balance ability. Improved scores in this test denote enhanced proprioception, cognition, and muscular strength. In this study, hemorrhage was induced in white rats at the bottom of the striatum, and the group with a steady single exercise and the group with alternate exercises were compared.

The beam-walking test is a test used mainly to check the ability of the upper and lower limbs in studies using rat models. The beam-walking test is evaluated from 0 to 6 , and the closer the 0 score, the better the performance, and the closer the 6 score, the lower the performance, and the performance of the balance, the upper and lower extremities coordination, and the feedback of the senses are evaluated. In this study, the performance of $4.53 \pm 0.52$ in the experimental group and $4.80 \pm 0.41$ in the control group are statistically significantly increased in the experimental group, but a future assessment of whether there is clinical significance should be made due to the small difference in the scores.

The expression of BDNF confirms the histological change, and if the expression of BDNF is high, it can be expected that there is a high possibility of neural regeneration and can have a good effect on the prognosis of intracerebral hemorrhagic patients. In this study, the experimental group showed statistical significance with the expression of BDNF 107.08 \pm 1.85 and the control group $104.97 \pm 0.85$. However, the difference is small, so it is important to check for clinical significance, as is the case with beam-working tests.

In the future, studies that increase the duration of the study and how to shorten the shift period of motion will require studies that generalize the results of this study. Based on the results of this study, it is hoped that a study will be conducted to generalize the results of the study by comparing the before and after comparison between groups and the amount of change over time.

However, this study had some limitations. First, the swimming exercise might have produced better results than wheel running due to the absence of weight bearing. Second, because of the prolonged training period, it was unclear whether the recovery of motor skills and neurotrophic substances following cerebral hemorrhage resulted from alternate training only.

\section{Conclusion}

The present study investigated the effects of alternate exercises on the recovery of ischemic cerebral infarction-induced white rats. The alternate training group's lowered scores in the beam walking test and increases in the BDNF signified that alternate exercises can have better effects on the recovery of nerves and motor skills than can a single exercise. Therefore, this study proposes this alternate pattern of physical exercises as a useful intervention for patients with intracerebral hemorrhage. 


\section{Acknowledgements}

This work was supported by the National Research Foundation of Korea (NRF) grant funded by the Korea government (MSIT) (No-2019R1F1A1057731).

\section{References}

[1] A. Qureshi, A. Mendelow, and D. Hanley, “Intracerebral hemorrhage,” Lancet, vol.373, no.9675, pp.16321644, (2009) DOI: 10.1016/S0140-6736(09)60371-8

[2] C. MacLellan, K. Langdon, K. Churchill, S. Granter-Button, and D. Corbett, "Assessing cognitive function after intracerebral hemorrhage in rats," Behavioural Brain Research, vol.198, no.2, pp.321-328, (2009) DOI: 10.1016/j.bbr.2008.11.004

[3] S. Mayer and F. Rincon, "Treatment of intracerebral hemorrhage," Lancet Neurology, vol.4, no.10, pp.662672, (2005)

[4] H. Eda, S. Sato, Y. Sasaki, A. Adachi, and M. Ghazizadeh, "Ischemic damage and subsequent proliferation of oligodendrocytes in hippocampal CA1 region following repeated brief cerebral ischemia," Pathobiology, vol.76, no.4, pp.204-211, (2009) DOI: 10.1159/000218337

[5] J. Jia, Y. Hu, Y. Wu, G. Liu, H. Yu, O. Zheng, D. Zhu, C. Xia, and Z. Cao, "Pre-ischemic treadmill training affects glutamate and gamma aminobutyric acid levels in the striatal dialysate of a rat model of cerebral ischemia," Life Sciences, vol.84, no.15-16, pp.505-511, (2009) DOI: 10.1016/j.lfs. 2009.01.015

[6] C. Fox, L. Ramig, M. Ciucci, S. Sapir, D. McFarland, and B. Farley, "The science and practice of LSVT/LOUD: neural plasticity-principled approach to treating individuals with Parkinson disease and other neurological disorders," Speech Language Pathology, vol.27, no.4, pp.283-299, (2006) DOI: 10.1055/s-2006955118

[7] M. Voss, R. Prakash, K. Erickson, C. Basak, L. Chaddock, J. Kim, H. Alves, S. Heo, A. Szabo, S. White, T. Wojcicki, E. Mailey, N. Gothe, E. Olson, E. McAuley, and A. Kramer, "Plasticity of brain networks in a randomized intervention trial of exercise training in older adults," Front Aging Neuroscience, vol.2, no.32, (2010) DOI: 10.3389/fnagi.2010.00032

[8] L. Ma, B. Wang, S. Narayana, E. Hazeltine, X. Chen, D. Robin, P. Fox, and J. Xiong, "Changes in regional activity are accompanied with changes in inter-regional connectivity during 4 weeks motor learning," Brain Research, vol.1318, pp.64-76, (2010) DOI: 10.1016/j.brainres.2009.12.073

[9] S. Jang, "The effects of familiar exercise and novel exercise on brain recovery after intracerebral hemorrhage in rats," $\mathrm{Ph}$. D. dissertation, Rehabilitation Medicine department, Daegu University, Gyeongsan-si, Gyeongsangbuk-do, Republic of Korea, (2012)

[10] K. Cowansage, J. LeDoux, and M. Monfils, "Brain-derived neurotrophic factor: a dynamic gate keeper of neural plasticity," Current Molecular Pharmacology, vol.3, no.1, pp.12-29, (2010) DOI: 10.2174/1874467211003010012

[11] G. Nagappan and B. Lu, "Activity-dependent modulation of the BDNF receptor TrkB: mechanisms and implications," Trends in Neurosciences, vol.28, no.9, pp.464-471, (2005) DOI: 10.1016/j.tins.2005.07.003

[12] G. Brooks and T. White, "Determination of metabolic and heart rate responses of rats to treadmill exercise," Journal of applied physiology: respiratory, environmental and exercise physiology, vol.45, no.6, 1009-1015, (1928) DOI: 10.1152/jappl.1978.45.6.1009

[13] K. Chu, J. Eng, A. Dawson, J. Harris, A. Ozkaplan, and S. Gylfadottir, "Water-based exercise for cardiovascular fitness in people with chronic stroke: a randomized controlled trial," Archives of Physical Medicine and Rehabilitation, vol.85, no.6, pp.870-874, (2004) DOI: 10.1016/j.apmr.2003.11.001

[14] D. Kwon, "Effects of swimming training on immune function of growing rats fed a high-fat diet," Journal of the Human-Environment System, vol.8, no.1, pp.13-18, (2005) DOI: 10.1618/jhes.8.13 
[15] M. Greenberg, B. Xu, B. Lu, and B. Hempstead, "New insights in the biology of BDNF synthesis and release: implications in CNS function," Journal of Neuroscience, vol.29, no.41, pp.12764-12767, (2009) DOI: 10.1523/JNEUROSCI.3566-09.2009

[16] R. Wang, Y. Yang, and S. Yu, "Protective effects of treadmill training on infarction in rats," Brain Research (2001), vol.922, no.1, pp.140-143, (2001) DOI: 10.1016/s0006-8993(01)03154-7

[17] S. Allen and D. Dawbarn, "Clinical relevance of the neurotrophins and their receptors," Clinical Science (London, England), vol.110, no.2, pp.175-191, (2006) DOI: 10.1042/CS20050161

[18] C. Bramham and E. Messaoudi, "BDNF function in adult synaptic plasticity: the synaptic consolidation hypothesis," Progress in Neurobiology, vol.76, no.2, pp.99-125, (2005) DOI: 10.1016/j.pneurobio.2005.06.003

[19] M. Sasaki, C. Radtke, A. Tan, P. Zhao, H. Hamada, K. Houkin, O. Honmou, and J. Kocsis, "BDNFhypersecreting human mesenchymal stem cells promote functional recovery, axonal sprouting, and protection of corticospinal neurons after spinal cord injury," Journal of Neuroscience, vol.29, no.47, pp.14932-14941, (2009) DOI: 10.1523/JNEUROSCI.2769-09.2009 